Research Article

\title{
Comparative study of the efficacy and safety of topical sertaconazole versus topical terbinafine in the treatment of dermatophytoses in a tertiary care centre
}

\author{
Mrinal Borgohain, Elango P*, Darling Chellathai
}

Department of Pharmacology, Sri Ramachandra Medical College and Research Institute Sri Ramachandra University, Chennai-600116, India

Received: 07 March 2016

Revised: 11 May 2016

Accepted: 12 May 2016

*Correspondence to:

Dr. Elango P,

Email: drpelango@yahoo.com

Copyright: (C) the author(s), publisher and licensee Medip Academy. This is an openaccess article distributed under the terms of the Creative Commons Attribution NonCommercial License, which permits unrestricted noncommercial use, distribution, and reproduction in any medium, provided the original work is properly cited.

\begin{abstract}
Background: Dermatophytes are superficial fungal infection termed dermatophytosis, diagnosed by direct microscopy and confirmed with culture and treated with antifungal agents by both topical and systemic therapy. The objective of this study was to compare the efficacy of topical sertaconazole with topical terbinafine applied twice daily in reducing the size and severity of the lesions.
\end{abstract}

Methods: Two groups of patients, the topical sertaconazole nitrate $2 \%$ cream (group 1) and the topical terbinafine hydrochloride 1\% cream (group 2) were treated twice daily for 2 weeks and followed after 4 weeks. Skin scrapings were taken to confirm the diagnosis, to assess the efficacy and potency of the study drugs. The statistical analysis was performed using statistical package for Social Sciences. Assessment of the groups was analysed using Chi-square test. Baseline demographic data was analysed using ANOVA.

Results: At the end of first week, sertaconazole group showed significant changes in pruritis ( $\mathrm{p}$ value 0.045 ) as compared to terbinafine group ( $\mathrm{p}$ value $<0.05$ ) and no severe symptoms of erythema in both grups. After 2 weeks, resolution of vesicle was seen higher in terbinafine group. But after 'follow-up phase', all patients showed absence of vesicles and negative mycological assessment.

Conclusions: Topical sertaconazole nitrate was better in reducing the severity of pruritis and achieving faster mycological cure compared to terbinafine and found to possess additional anti-pruritic and anti-inflammatory action.

Keywords: Dermatophytosis, Sertaconazole, Terbinafine, Pruritis, Erythema, Vesicles

\section{INTRODUCTION}

Dermatophytes are a group of taxonomically related fungi. ${ }^{1}$ Superficial infection caused by a dermatophyte is termed dermatophytosis or ringworm. They are all moulds belonging to three asexual genera: microsporum, trichophyton and epidermophyton. The common species which causes human infection include $T$. rubrum, $T$. mentagrophytes, $T$. tonsurans, $T$. violaceum, $T$. schonleinii, E. floccosum, M. audouini etc. Depending upon the site of infection, dermatophyte infection can be classified as tinea corporis (body), tinea cruris (groin), tinea capitis (head), tinea pedis (feet), tinea manuum (hand), tinea unguium (nail), tinea barbae (beard) etc. ${ }^{2}$ The most common factors predisposing to fungal infection still remain poor personal hygiene, immune status and associated illness. ${ }^{3}$ The two most important methods used to diagnose dermatophytosis are direct microscopy and isolation of the specific species through culture. Dermatophyte infections are treated with antifungal agents like polenes, allylamines and azoles by both topical and systemic antifungal therapy. Its prevention and control must take into account the etiological agent, area invaded and the source of 
infection. Individual care and hygiene are important measures of prevention.

\section{Rationale of the study}

Two pilot studies so far in India have compared the efficacies of terbinafine hydrochloride $1 \%$ cream and sertaconazole nitrate $2 \%$ cream. Choudhary et al. compared the efficacy of topical terbinafine hydrochloride $1 \%$ cream with topical sertaconazole nitrate $2 \%$ cream in 38 patients. ${ }^{4}$ According to this study sertaconazole nitrate $2 \%$ cream was as effective as terbinafine hydrochloride $1 \%$ cream though terbinafine hydrochloride $1 \%$ cream has higher rates of complete cure at the end of 2 weeks as compared to sertaconazole nitrate $2 \%$ cream. Jerajani, et al. compared three drugs sertaconazole nitrate $2 \%$, terbinafine hydrochloride $1 \%$ and luliconazole $1 \%$ cream in 83 patients and reported that sertaconazole was better than terbinafine and luliconazole in relieving signs and symptoms during study and follow up period. ${ }^{5}$ Since, it was not possible to come to a strong conclusion regarding the efficacy and safety of topical sertaconazole nitrate $2 \%$ cream, we undertook this study with a larger sample size with post treatment follow to check for possible recurrence of lesions which was not done in previous studies.

The objective of this study was to determine the efficacy of topical sertaconazole applied twice daily with topical terbinafine applied twice daily in reducing the size and severity of lesions.

\section{METHODS}

The design of this study was a randomized controlled, two group parallel arm, open labeled, single center study with active comparator. The study protocol was reviewed and approved by institutional ethics committee and informed consent was obtained from all study participants before performing any procedure related to this study.

\section{Inclusion criteria}

- $\quad$ Subjects are males or non-pregnant females

- Age between 18 years and 70 years willing and able to give written informed consent

- With the clinical diagnosis of tinea corporis and tinea cruris infections

\section{Exclusion criteria}

- Subjects with the clinical diagnosis of tinea pedis/manum, tinea capitis and tinea barbae

- Subjects who had received topical or oral antimycotics either one or four weeks prior to the initiation of the study respectively

- Subject has history of hypersensitivity to the study drugs
- Subject with immune-compromised status, superadded bacterial infection or pregnant or lactating women.

- Patients with any other co morbid illness

- Patients who are not willing to give informed consent.

\section{Study procedure}

Out of 195 patients screened, 45 patients were excluded due to screening failure and remaining 150 patients were randomly assigned to two groups: topical sertaconazole nitrate group and topical terbinafine hydrochloride group, each consisting of 75 patients. Among 150 patients randomized, 123 patients completed the study while 27 patients discontinued (14 patients in sertaconazole nitrate arm and 13 patients in terbinafine hydrochloride arm). 13 patients in sertaconazole nitrate arm and 11 patients in terbinafine hydrochloride arm were lost to follow up during the follow up visits. 1 patient in both the arms withdrew consent due to personal reasons. 1 patient in terbinafine hydrochloride arm was withdrawn from the study as the patient was not able to tolerate the drug due to hypersensitivity. The flow of study participants is shown in (Figure 1) and gender distribution is shown in (Figure 2).

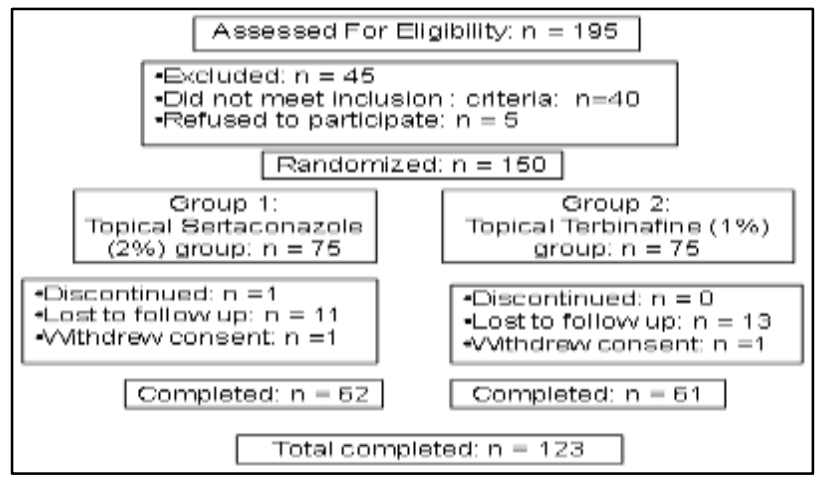

Figure 1: Study participants flow charts.

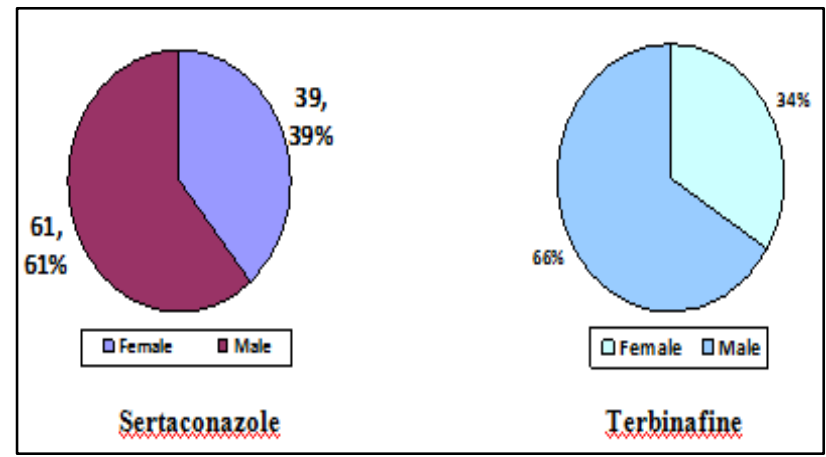

Figure 2: Gender distribution.

Baseline investigations were done before prescribing the test drugs. The patients in one group received topical sertaconazole nitrate $2 \%$ cream twice daily and the other 
group received topical terbinafine hydrochloride $1 \%$ cream twice daily. The study treatment duration in both the groups was 2 weeks. Post treatment follow up was done at $4^{\text {th }}$ week. There were two scheduled visits during the study starting with baseline visit, then after $1^{\text {st }}$ week and $2^{\text {nd }}$ week and a follow up visit at 4 th week during the post treatment period ( 2 weeks after stopping the drug). Clinical and mycological assessment was done at baseline and after $1^{\text {st }}$ week and $2^{\text {nd }}$ week of the study. The study drug was purchased from whole sale stockiest (brand name: Onabet and Tyza, manufacturer: Glenmark and Abbott pharmaceuticals respectively) and supplied to the study participants during each scheduled visits along with compliance card. All the study participants were instructed to apply a thin layer of the study medication around the margin of the tinea lesions. Application to normal skin was to be avoided. Study medications should be applied after the skin has dried if the patient bathed or showered in the evening. The patients were also instructed to avoid exposure to excessive sunlight. During the study period other local or systemic medications for treatment of tinea were not permitted. In the event of local irritation, only liquid paraffin oil was permitted. Adverse event reporting during the study were noted

\section{Laboratory investigations}

Laboratory investigation was done during the baseline visit of the study period. Fasting blood glucose level and HbA1c levels were taken. Skin scrapings were taken in all four visits and mounted on $\mathrm{KOH}$ mount. ${ }^{5}$ The slides were examined under microscope, first under low power (10x) and then under high power (40x) objective, for presence of thin filamentous forms (hyphae).

\section{Study end points}

I. Efficacy end points.

i. Atleast $70 \%$ improvement in clinical symptoms and signs of tinea lesions relative to baseline visit.

ii. To achieve Mycological cure of atleast $80 \%$ relative to baseline visit.

- Clinical assessment was based on the proportion of patients with symptoms and signs of tinea lesions namely pruritis and erythema are graded depending on intensity as none: 0 , mild: 1 , moderate: 2 and severe: 3 and the vesicles are observed as present and absent. $^{6}$

- Mycologic assessment was based on $10 \% \mathrm{KOH}$ mounting for dermatophytes of the skin scrapings. ${ }^{5}$

II. Grading scales for assessment of pruritis and erythema severity.

i. Pruritis grading scale ${ }^{7}$

- The pruritus grading system (PGS) score for each patient was based on: distribution, frequency, severity of itch and quality of sleep as shown below. Each patient's itch grade was calculated as the sum of the individual scores as:

Mild grade: if total score was between 0 and 5 . Moderate grade: if total score was between 6 and 11 . Severe grade: if total score was between 12 and 19

ii. Erythema grading scale

- Erythema is defined as abnormal redness of skin. The following grading scale was used in our study.

\begin{tabular}{|lll|}
\hline Severity & Score & Clinical feature \\
\hline None & 0 & No Erythema \\
\hline Mild & 1 & Slight pinkness present \\
\hline Moderate & 2 & $\begin{array}{l}\text { Definite redness, easily } \\
\text { recognized }\end{array}$ \\
\hline Severe & 3 & Intense redness \\
\hline
\end{tabular}

\section{Safety End Points}

- Incidence of adverse events in both groups.

\section{Statistical analysis}

The statistical analysis was performed using SPSS (Statistical package for social sciences) for windows version 16. Demographic data (age, sex, etc.) and baseline patient characteristics were summarized by treatment groups using descriptive statistics to assess the difference if any between them. Mean and standard deviation was provided for continuous data, while absolute frequencies and percentage were provided for categorical data. $\mathrm{P}$ value $<0.05$ was considered statistically significant. Mycological assessment and clinical assessment, within and between the groups were analysed using chi-square test. Baseline demographic data was analysed using ANOVA. All the analyses were based on modified intention to treat (ITT) principle. Modified ITT population included all the study participants who had completed at least one follow- up visit.

\section{RESULTS}

\section{Efficacy results}

The percentage of patients showing base line symptoms of pruiritis in both groups is shown in (Figure 3). The percentage of patients showing erythema severity in both groups at base line is shown (Figure 7). As shown in (Figures 4-6 and 8-10), there were considerable improvements in clinical symptoms in both the treatment groups during each follow up visit. The percentage of improvement for pruritis, erythema and appearance of the vesicles in sertaconazole group were $91.80 \%, 91.79 \%$ and $98.4 \%$ at the end of treatment phase and $96.7 \%, 98.4 \%$ and $100 \%$ at follow up respectively (Figure 12) and the 
percentage of improvement for pruritis, erythema and appearance of the vesicles in terbinafine group were $79 \%$, $85.5 \%$ and $100 \%$ at the end of treatment phase and $93.5 \%$, $90.3 \%$ and $100 \%$ at follow up respectively (Figure 13). The reduction in pruritis was significantly more in sertaconzole $(91.8 \%)$ group compared to terbinafine $(79 \%)$ group $(\mathrm{p}<0.05)$ at the end of treatment phase.

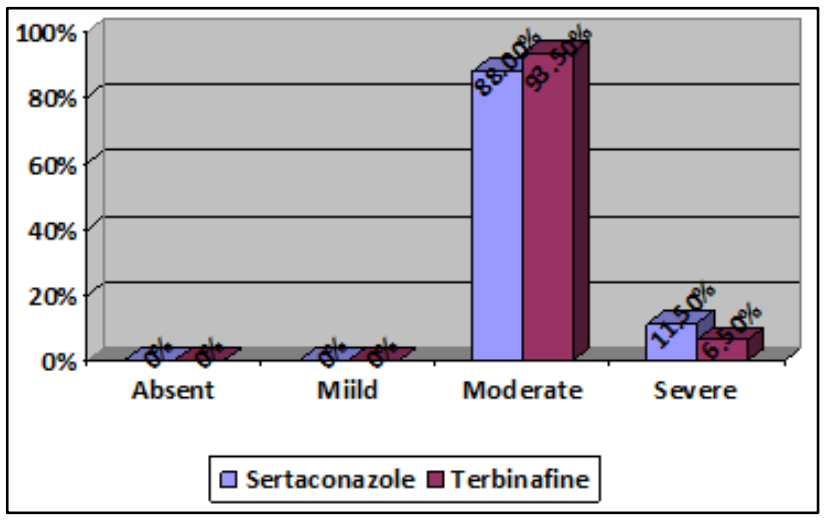

Figure 3: The percentage of patients showing base line symptoms of pruiritis.

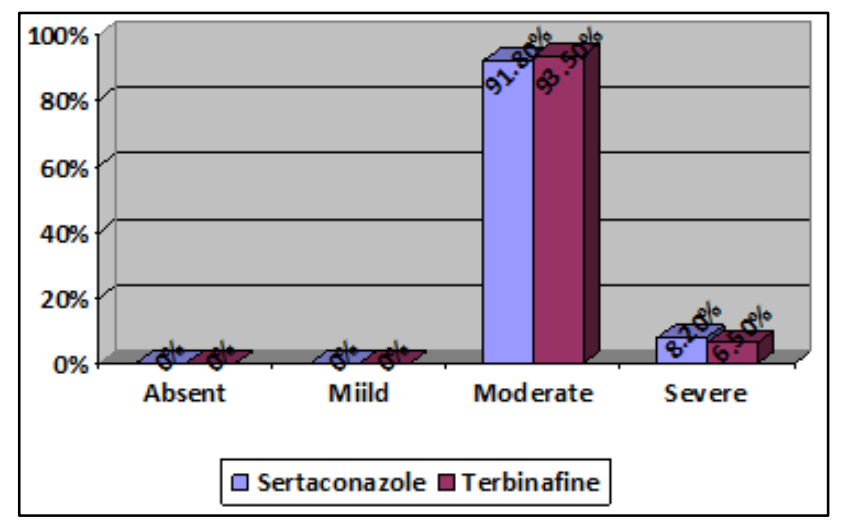

Figure 4: The percentage of patients showing symptoms of pruiritis after 1 week.

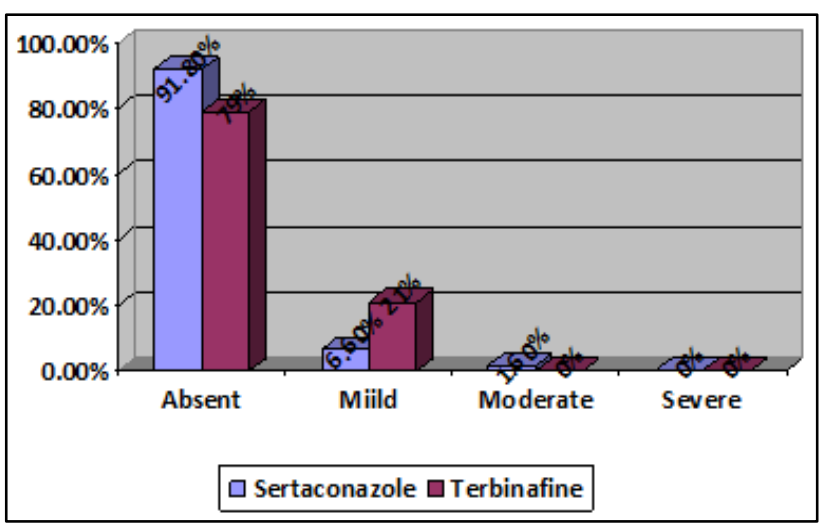

Figure 5: The percentage of patients showing symptoms of pruiritis after 2 weeks.

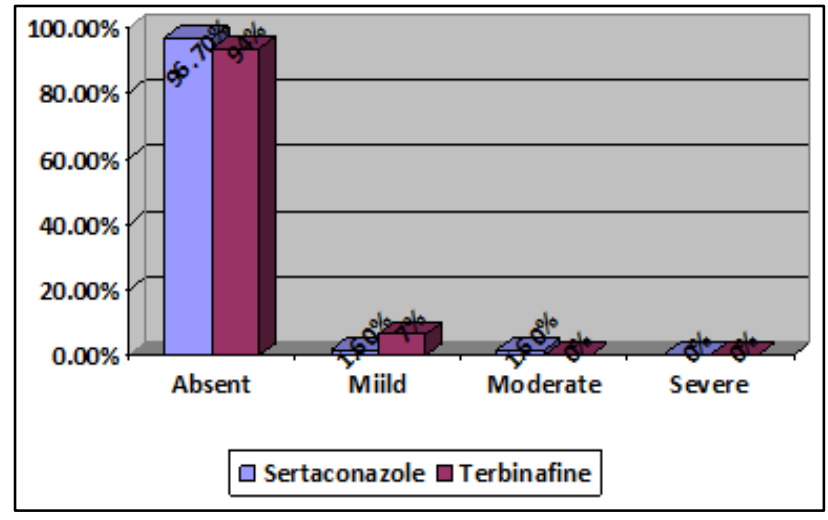

Figure 6: The percentage of patients showing symptoms of pruiritis the end of 4 weeks (follow up Phase).

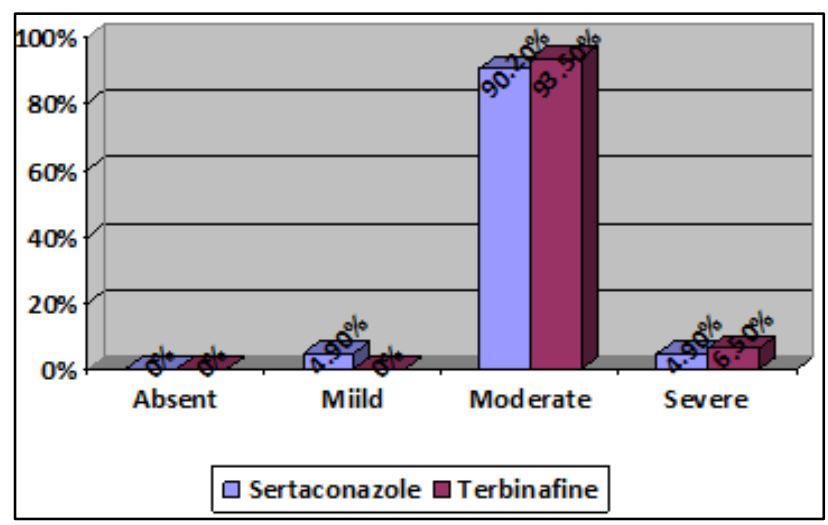

Figure 7: The percentage of patients showing erythema severity in both groups at base line.

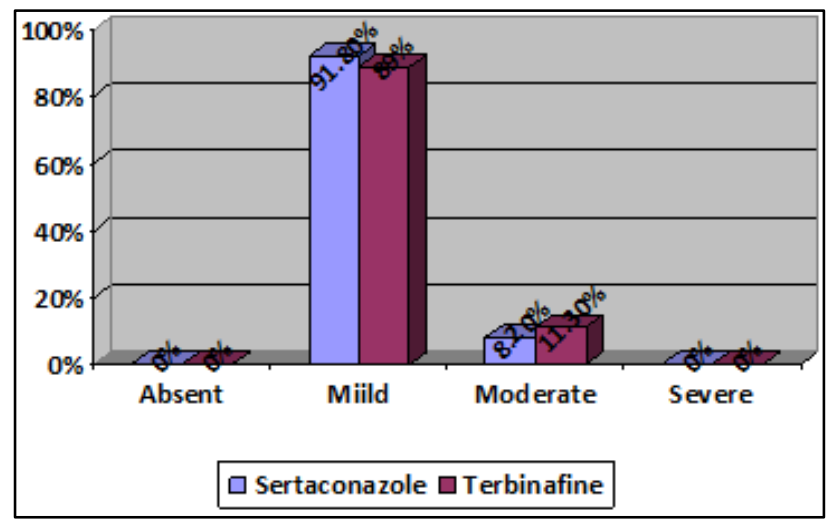

Figure 8: The percentage of patients showing improvement of erythema severity in both groups at the end of 1 week.

\section{Mycological assessment}

At baseline all patients had positive $\mathrm{KOH}$ test for Dermatophytes in both the groups. After the end of first week, mycological resolution was seen in higher proportion of patients in the sertaconazole group $(91.8 \%)$ compared to terbinafine group $(58.1 \%)$. The significance 
was 0.001 ( $\mathrm{p}$ value $<0.05)$ which is highly significant. At end of 'Treatment Phase' and 'follow-up' phase, all patients showed negative mycological assessment (negative $\mathrm{KOH}$ test). Since this is a qualitative data chi square test was done to analyse the data.

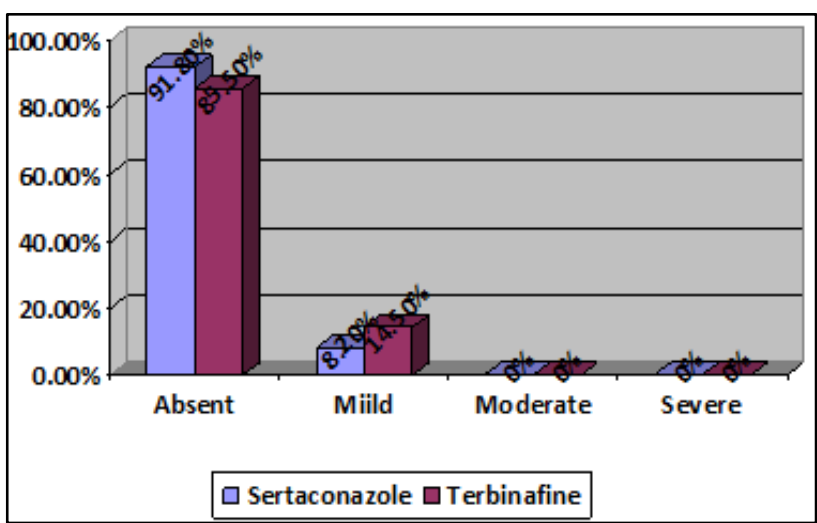

Figure 9: The percentage of patients showing improvement of erythema severity in both groups at the end of 2 weeks.

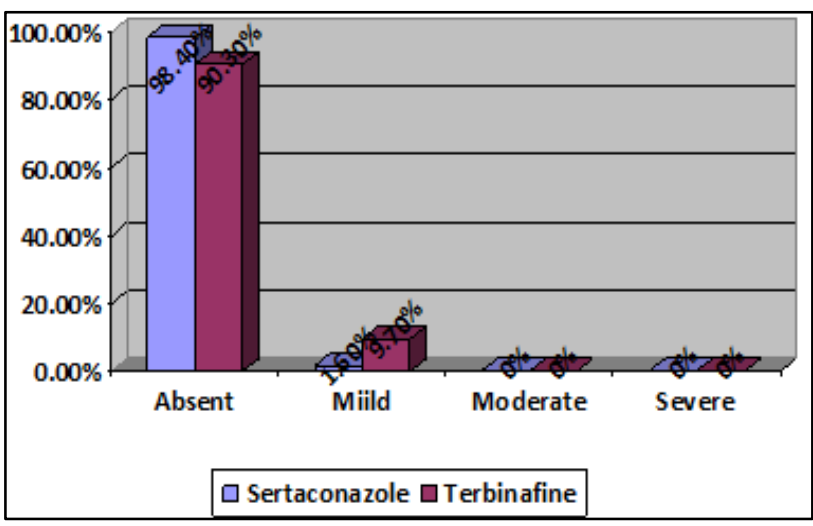

Figure 10: The percentage of patients showing improvement of erythema severity in both groups at the end of 4 weeks (follow up phase).

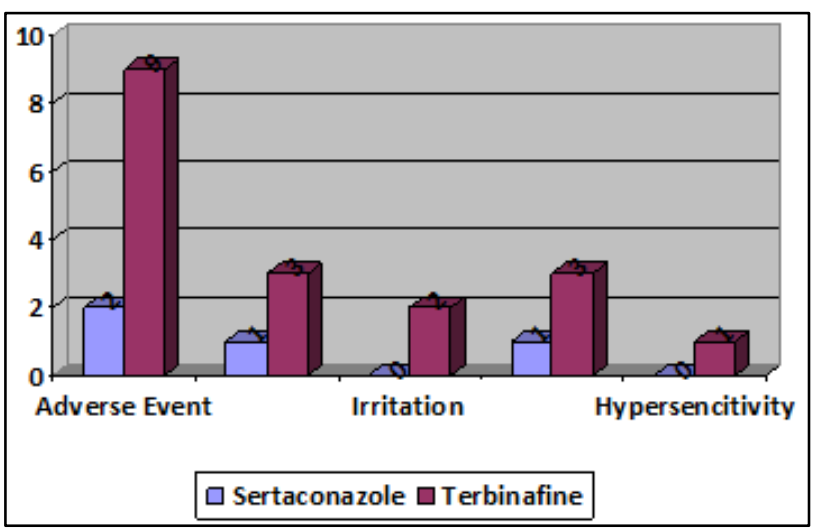

Figure 11: Incidence of adverse events in both groups during 4 weeks of study period.

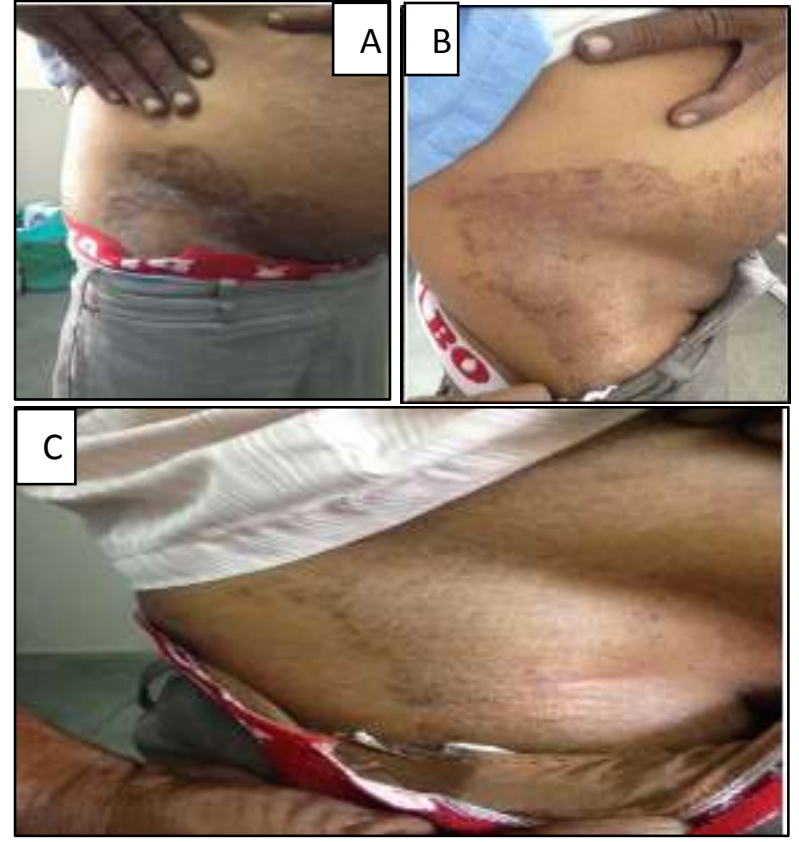

Figure 12: Treatment with sertaconazole $2 \%$ cream. A) Improvement in tinea corporis lesion with topical sertaconazole $2 \%$ cream (at baseline); B) improvement in tinea corporis lesion with topical sertaconazole $2 \%$ cream (at end of $1^{\text {st }}$ week); C) improvement in tinea corporis lesion with topical sertaconazole $2 \%$ cream (at end of $2^{\text {nd }}$ week).
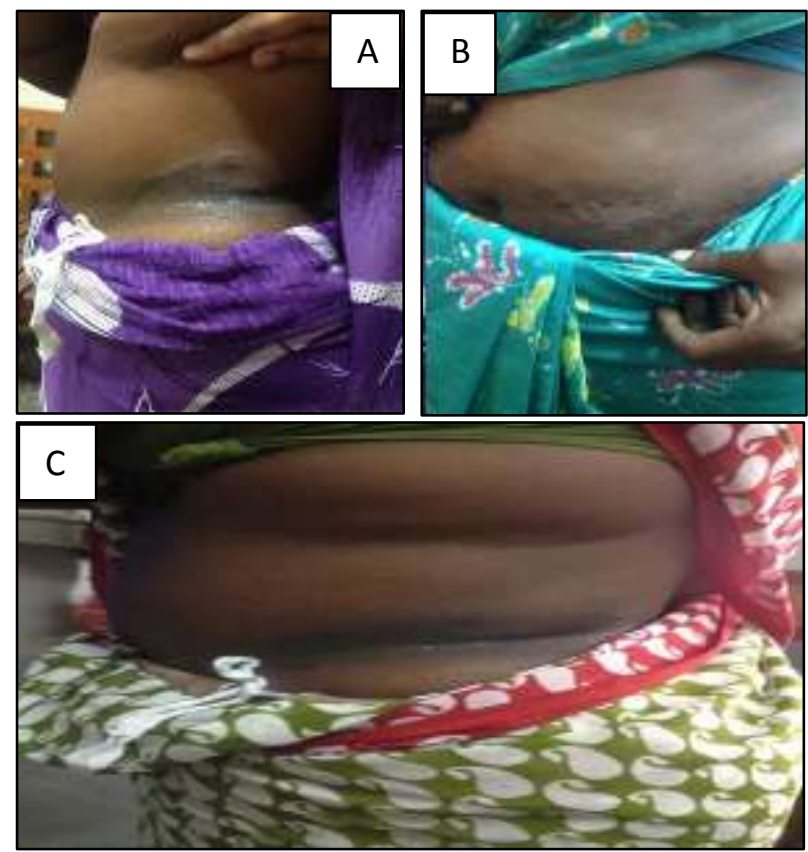

Figure 13: Treatment with terbinafine $1 \%$ cream. A) Improvement in tinea corporis lesion with topical terbinafine $1 \%$ cream (at baseline); B) Improvement in tinea corporis lesion with topical terbinafine $1 \%$ cream (at the end of 1st week); C) Improvement in tinea corporis lesion with topical terbinafine $1 \%$ cream (at the end of 2 nd week). 


\section{DISCUSSION}

The present study evaluated the efficacy and safety of topical sertaconazole nitrate $2 \%$ with topical terbinafine hydrochloride $1 \%$ in the treatment of dermatophytosis. This study is in accordance with the study conducted by Mohanty JC et al which mentions that the incidence of dermatophytosis in Orissa is more common in the second to fourth decade of life. ${ }^{8}$ Most of the study patients were males in both group A and group B which coincide with the previous studies. According to Sen SS et al males are more likely to present with tinea cruris than females. ${ }^{9}$ However no statistically significant difference was observed between both the groups in the mean percent change with erythema, vesicle and desquamation from baseline to 2 weeks of therapy or follow up. Sertaconazole also has additional anti-pruritic and antiinflammatory action. ${ }^{10-12}$ This antipruritic and antiinflammatory property of sertaconazole is due to its ability to reduce histamine release and several other proinflammatory cytokines including PGE2. This is comparable to the pilot study conducted by Choudhury SV et al. ${ }^{11,4}$

A study by Thaker et al has shown that sertaconazole produced $90 \%$ mycological clearance at the end of 2 weeks. ${ }^{13}$ In our study $91.8 \%$ of mycological clearance is seen with sertaconazole $(\mathrm{p}<0.05)$ compared to terbinafine which showed mycological clearance of $58.1 \%$ at the end of 1 week. However both the drugs showed complete mycological clearance of $100 \%$ by the end of 2 weeks.

During our entire study period the study drugs were well tolerated in both the groups as shown in (Figure 11). However the number of patients who reported adverse effects was comparatively more in terbinafine $(14.5 \%)$ group than sertaconzole $(3.2 \%)$ group. Hypersensitivity, burning sensation, localised erythema and excoriation of skin was noted and necessary treatment after consultation with senior dermatologist was given sertaconzole nitrate $2 \%$ cream showed better reduction in clinical symptoms of tinea lesions than that of terbinafine hydrochloride $1 \%$ cream.

Sertaconazole therefore produced a better and faster mycological cure compared to terbinafine at the end of 1 week which was statistically significant.

\section{CONCLUSION}

To conclude, topical sertaconazole nitrate $2 \%$ was superior to topical terbinafine hydrochloride $1 \%$ in resolution of clinical symptoms and improving PGA grading in tinea lesions. Topical sertaconazole nitrate was better in reducing the severity of pruritis and achieving faster mycological cure compared to terbinafine. This is because sertaconazole possess additional anti-pruritic and anti-inflammatory action apart from its potent anti-fungal action. However the severity of reducing erythema, vesicle and desquamation were almost equivalent in both the drugs. The safety profile of sertaconazole was also better compared to terbinafine.

Thereby, our study proves that topical sertaconazole nitrate $2 \%$ is a safe, effective, better and well tolerated therapy than topical terbinafine hydrochloride $1 \%$ for patients with dermatophytosis.

Funding: No funding sources

Conflict of interest: None declared

Ethical approval: The study was approved by the Institutional Ethics Committee

\section{REFERENCES}

1. Calvin OM, Thomas JL. Eczema, psoriasis, cutaneous infections, acne and other common skin disorders. In: Fauci, Braunwald, Kasper, Hauser, Longo, Jameson, Loscalzo, editors. Harrison's Principles of Internal Medicine. $17^{\text {th }}$ ed, New York:McGraw-Hill; 2008:1:318.

2. Rippon JW. Superficial infection. In: Chapter 6, In Medical Mycology: The Pathogenic fungi and The Pathogenic Actinomycetes, $3^{\text {rd }}$ edition, W.B. Saunders Co. Philadelphia; 1988:154-159.

3. Banerjee M, Ghosh AK, Basak S, Das KD, Gangopadhyay DN. Comparative evaluation of effectivity and safety of topical topical amorolfine and clotrimazole in the treatment of tinea corporis. Indian Journal of Dermatology. 2011;56(6):657-62.

4. Choudhary SV, Bisati S, Singh AL, Koley S. Efficacy and safety of terbinafine hydrochloride $1 \%$ cream versus sertaconazole nitrate $2 \%$ cream in tinea corporis and tinea cruris: a comparative therapeutic trial. Indian Journal of Dermatology. 2013;58(6):457-60.

5. Ilkit M. Demirhindi H. Asymptomatic dermatophyte scalp carriage: laboratory diagnosis, epidemiology and management Mycopathologia. 2008;165:61-6.

6. Jerajani HR, Janaki C, Kumar S, Phiske M. Comparative assessment of sertaconazole, terbinafine, luliconazole cream in dermatophytosis; A Pilot Study. Indian Journal of Dermatology. 2013;58(1):34-38.

7. Al-Qarqaz F, Al-Aboosi M, Al-Shiyab D, Bataineh A. Using pruritus grading system for measurement of pruritus in patients with diseases associated with itch; J Med J. 2012;46(1):39-44.

8. Mohanty JC, Mohanty SK, Sahoo RC, Sahoo AS, Praharaj $\mathrm{CH}$. Incidence of dermtophytosis in Orissa. Indian J Med Microbiol. 1998:16:78-80.

9. Sen SS, Rasul ES. Dermatophytosis in Assam. Indian J Med Microbiol. 2006;24:77-8.

10. Carrillo-Muñoz AJ, Tur-Tur C, Bornay-Llinares FJ, Arévalo P. Comparative study of the in vitro antifungal activity of bifonazole, naftifine and sertaconazole against yeasts. J Chemother. 1999;11(3):187-90.

11. Liebel F, Lyte P, Garay M, Babad J, Southall MD. Anti-inflammatory and anti-itch activity of topically 
applied sertaconazole nitrate. Arch Dermatol Res. 2006;298(4):191-9.

12. Agut J, Tarrida N, Sacristan A, Ortiz JA. Antiinflammatory activity of topically applied sertaconazole nitrate. Meth Find Exp Clin Pharmacol 1996;18(4):233-4.
13. Thaker SJ, Mehta DS, Shah HA, Dave JN, Mundhava SG. A comparative randomized open label study to evaluate efficacy, safety and cost effectiveness between topical $2 \%$ sertaconazole and topical $1 \%$ butenafine in tinea infections of skin. Indian Journal of Dermatology. 2013;58(6):451-6.

Cite this article as: Borgohain $\mathrm{M}$, Elango $\mathrm{P}$, Chellathai D. Comparative study of the efficacy and safety of topical sertaconazole versus topical terbinafine in the treatment of dermatophytoses in a tertiary care centre. Int J Basic Clin Pharmacol 2016;5:855-61. 